\title{
¿Qué nos aporta el modelo de patrones de aprendizaje para el diseño de acciones formativas?
}

What Does the Learning Patterns Model

Contribute to the Design of

Educational Actions?

O que o modelo de padrões de aprendizagem

contribui para o desenho de

ações de treinamento?

\section{Carmen Ruiz Bueno* iD orcid.org/0000-0003-3694-3150 \\ Jordi García-Orriols** (iD orcid.org/0000-0003-2655-1081}

doi: 10.17227/rce.num77-9527

Para citar este artículo: Ruiz-Bueno, C. y García-Orriols, J. (2019). ¿Qué nos aporta el modelo de patrones de aprendizaje para el diseño de acciones formativas? Revista Colombiana de Educación, 77, 321-341. doi: 10.17227/rce.num77-9527

\section{(c) $)$ (1) (8)}

* Profesora titular del Departamento de Pedagogía Aplicada. Universitat Autònoma de Barcelona. Correo electrónico: carmen.ruiz.bueno@uab.cat

** Colaborador del grupo de investigación Pafiu, Universitat Autònoma de Barcelona. Correo electrónico: pafiu.uab@gmail.com 


\section{Resumen}

Los grandes y veloces cambios que vive la sociedad actual invitan a cuestionarse y a reflexionar sobre los modelos educativos que se están llevando a cabo para hacer frente a estas nuevas realidades. De este modo, se presenta la necesidad de activar procesos de análisis del sector educativo, no solamente de las etapas de educación superior sino haciendo hincapié en la base de esta, en Educación Primaria. En tal sentido, este artículo de reflexión establece bases y principios para las acciones formativas, a partir de resultados de un estudio previo sobre el modelo de Patrones de Aprendizaje de Vermunt (1998) en el que participaron un total de 292 alumnos catalanes de Educación Primaria. Estas nuevas líneas de actuación están orientadas al fomento de un aprendizaje más reflexivo, crítico y autorregulado mediante la cooperación entre alumnos, teniendo en cuenta sus características individuales en lo que a su proceso de aprendizaje se refiere. A raíz de ello, es cada vez más necesario desde los centros educativos crear estos entornos de aprendizaje con el fin de potenciar futuros ciudadanos más creativos, consecuentes y que promuevan acciones de cambio orientadas a la mejora social.El artículo se estructura en una primera parte en la reflexión sobre los patrones de aprendizaje y en una segunda y tercera donde se apuntan las ideas claves acerca de los fundamentos y principios de las acciones formativas para su posterior discusión con el modelo de Vermunt (1998)

\section{Palabras clave}

estrategias de formación; autodesarrollo; necesidades de la infancia; aprendizaje cooperativo

\section{Keywords}

training actions; self-regulation; learning patterns; childhood; cooperative learning
Nowadays societies are suffering several changes which invite to think and reflect on the educational models that are established currently to face these new realities. Thus, societies need to analyse processes regarding the educational area, and not only the stages of higher education but emphasizing its basis, that is, Primary Education. In this sense, this article presents a set of training actions derived from a previous study. This study is based on the learning patterns model of Vermunt (1998) in which a total of 292 catalan students of Primary Education participated. These new lines of action are aimed at fostering reflexive, critical and self-regulated learning through cooperation among students, taking into account their individual learning processes. As a result, it is necessary that schools create these types of learning contexts in order to promote more creative and consistent future citizens capable of promoting

\section{Resumo}

As grandes e rápidas mudanças que a sociedade atual está vivendo nos convida a questionar e refletir sobre os modelos educacionais que estão sendo utilizados para enfrentar essas novas realidades. Assim, há a necessidade de ativar processos de análise do setor educação, não apenas das etapas do ensino superior, mas enfatizando as bases deste, no Ensino Fundamental. Nesse sentido, este artigo de reflexão estabelece bases e princípios para as ações formativas, com base nos resultados de um estudo anterior sobre o modelo de Padröes de Aprendizagem de Vermunt (1998), no qual participaram 292 estudantes de Educação Básica da Catalunha. Essas novas linhas de ação visam fomentar uma aprendizagem mais reflexiva, crítica e auto-regulada por meio da cooperação entre os alunos, levando em conta suas características individuais em termos de seu processo de aprendizagem. Como resultado, é cada vez mais necessário que as escolas criem estes ambientes de aprendizagem de modo a capacitar futuros cidadãos mais criativos, consequentes e que promovem ações de mudança voltadas para o aprimoramento social. O artigo está estruturado em uma primeira parte na reflexão sobre os padrões de aprendizagem e numa segunda e terceira, onde as idéias-chave sobre os fundamentos e princípios das ações de formação são apontadas para sua posterior discussão com o modelo de Vermunt (1998).

\section{Palavras-chave}

estratégias de treinamento autodesenvolvimento; necessidades das crianças; aprendizagem cooperativa 


\section{Introducción}

En este artículo se discute el modelo de patrones de aprendizaje y sus aportaciones para el diseño de acciones formativas que fomenten un aprendizaje autorregulado en el ámbito de la educación primaria.

Para ello, se describen las principales características del modelo de patrones de aprendizaje (Marambe, Vermunt y Boshuizen, 2012; Martínez-Fernández y Vermunt, 2015; Vermunt, 1998, 2005; Vermunt, Bronkhorst y Martínez-Fernández, 2014). Se analizan las bases, los fundamentos y principios de acción que deberían adoptar las medidas de formación encaminadas al desarrollo de un aprendizaje significativo, profundo y autorregulado. De igual modo, se exponen algunos resultados de los estudios de Martínez-Fernández, Galera-Bassach y García-Orriols (2017) y Martínez-Fernández, García-Ravidá, García-Orriols y Martí-Garbayo (2018) en relación con la identificación de los patrones de aprendizaje y otros factores (esfuerzo y rendimiento académico, autoestima y autoconcepto) junto con el diseño y desarrollo posterior de un conjunto de acciones formativas con base en el modelo (1998) en un contexto de educación primaria. Por último, se establecen los lineamientos metodológicos más significativos para la construcción de un modelo de acciones formativas, fundamentado en el marco conceptual y teórico de patrones de aprendizaje.

\section{Acerca de los patrones de aprendizaje}

Aprender hoy significa construir conocimiento a partir de distintas experiencias personales y sociales mediante la interacción con otros, en escenarios y situaciones muy diversas.

De igual forma, no se asume el aprendizaje como un proceso circunscrito a la institución escolar, sino como un proceso a lo largo de la vida en el cual se interconectan escenarios y contextos tanto formales como informales. Así, las escuelas dejan de ser escenarios primarios de enseñanza y aprendizaje (Bolívar, 2008; Burbules, 2012; Torrano, Fuentes y Soria, 2017), y pasan a constituir una parte integrante de una red compleja de instituciones, de contextos y de personas que interactúan para desarrollar procesos de aprendizaje mucho más significativos, situados, profundos e integrales (Biggs, 2010; Díaz Barriga, 2006; Ortega, 2017, Soler, 2006).

Asimismo, el aprendizaje se entiende como un proceso interactivo, socio-construido y mediado, que implica procesos cognitivos (vinculados con la motivación, la regulación y el procesamiento de información) y emocionales (vinculados a las creencias, los valores, las actitudes, etc.); 
influido por condiciones personales (como edad y género) y contextuales (Boekaerts y Cono, 2005; De la Barrera, Donolo y Rinaudo, 2010; Martínez-Fernández y García-Ravidá, 2012; Martínez-Fernández y Vermunt, 2015; Vermunt, Bronkhorst y Martínez-Fernández, 2014; Vermunt y Endedijk, 2011) que favorecen o no su desarrollo; condiciones relacionadas con la escuela, la familia, la sociedad, la cultura, el grupo de iguales u otros elementos del contexto.

En tal sentido, es oportuno tener como referente el modelo de patrones de aprendizaje de Vermunt $(1998,2005)$ para tomar decisiones sobre cómo enseñar y, sobre todo, cómo diseñar acciones formativas que potencien un aprendizaje más reflexivo-crítico y autorregulado.

El modelo en cuestión (Vermunt, 1998, 2005) establece dos ejes que se interrelacionan para la comprensión de los procesos de aprendizaje. Por un lado, el sujeto que aprende parte de un primer eje epistemológico de creencias (concepciones de aprendizaje) y motivaciones (orientación motivacional) que determinan un segundo eje de acción: el conjunto de acciones de aprendizaje (estrategias de procesamiento de la información) que se activan mediante un proceso regulador entre las formas de pensar y de revisar dicha actuación (estrategias de regulación). Además, estos ejes se ven influenciados por un conjunto de factores personales y contextuales que luego también determinarán ciertas formas de aprender (Vermunt, 2005).

Cabe destacar que el aporte más significativo del autor, en su modelo, radica en la idea de combinar las subcategorías de los factores mencionados, lo que da lugar a distintos patrones según se sitúen los alumnos, y cómo se caracterizan en cada uno de los factores.

Así, el modelo de Vermunt (1998) identifica cuatro patrones de aprendizaje en función de la combinación de las subcategorías:

» Patrón MD (meaning directed), caracterizado por una concepción del aprendizaje vinculado a la construcción y creación de conocimiento, a partir de una orientación motivacional intrínseca o interés personal, que activa el uso de estrategias reflexivo -críticas y de autorregulación mediante un procesamiento profundo de la información.

» Patrón AD (application directed), caracterizado por una concepción de aprendizaje basada en el uso del conocimiento a partir de una motivación vocacional que activa estrategias de autorregulación y regulación externa además de un procesamiento de la información centrado en situaciones concretas. 
» Patrón RD (reproduction directed), caracterizado por una concepción del aprendizaje basada en el incremento de conocimientos, motivación por los certificados y calificaciones, regulación externa y estrategias memorísticas en el procesamiento de la información.

» Patrón UD (undirected), caracterizado por una concepción del aprendizaje estimulado, orientación motivacional ambivalente y una carencia de estrategias de regulación y de procesamiento de la información.

Recientemente, Martínez-Fernández et al. han considerado de vital importancia la aplicación del modelo de patrones de aprendizaje en el contexto de la educación primaria, con la finalidad de articular acciones formativas adecuadas, pertinentes a la realidad y al contexto de la escuela y sus alumnos, para la articulación de propuestas metodológicas y evaluativas para el fomento de aprendizajes reflexivo-críticos y autorregulados. Aunque su estudio ha sido menor que en otros contextos y niveles educativos, trabajos de investigación como los de Berdonces Ballesteros (2015), Cladellas-Pallarès (2015), Cortés (2015), García-Martín (2014), Villar-López (2013) y Martínez-Fernández, Galera-Bassachs y García-Orriols (2017), así como los de Martínez-Fernández, García-Ravidá, García-Orriols y Martí-Garbayo (2018) constatan la identificación de dichos patrones (MD, $A D, R D$ y UD) en alumnos de estos niveles; y destacan la relevancia de tal identificación.

\section{Fundamentos de las acciones formativas para desarrollar patrones de aprendizaje autorregulados}

Los procesos de enseñanza y de aprendizaje orientados a estimular patrones de aprendizaje autorregulados deberían enfocarse hacia la creación de situaciones auténticas de aprendizaje en las que la motivación intrínseca, la participación activa, la colaboración y el pensamiento reflexivo-crítico se conviertan en ejes del proceso formativo, además de todas aquellas competencias ligadas a aprender a aprender (como por ejemplo: autonomía y cooperación).

Atendiendo a estas últimas, y como lo plantean Coll, Mauri y Rochera (2012) "aprender a aprender" no solo se refiere a un proceso que se desarrolla con la participación de los alumnos, sino que es más complejo, requiere de reflexión, construcción significativa del conocimiento y autorregulación. A su vez, dicho aprendizaje debe basarse en la reflexión, tanto individual como grupal, en la interacción con otros y en el uso de 
estrategias metacognitivas, centradas en un pensamiento anticipatorio, de planificación, concienciación, control, reacción y reflexión (Panadero y Alonso, 2014; Ruiz et al., 2018, Torre, 2007). Estas estrategias han de facilitar el aprendizaje, conjuntamente con estrategias motivacionales relacionadas con la dimensión emocional (sentimientos, estimaciones, juicios relativos a uno mismo y a las tareas de aprendizaje) (Efklides, 2009; Zimmerman Kitsantas y Campillo2005) y la contextual, que establece como factores influyentes el clima y las relaciones sociales en el contexto de la activación de procesos de enseñanza y aprendizaje (Boekaerts y Cascallar, 2006; Martínez-Fernández, 2002).

Será clave para crear un contexto estimulante, generador de pensamiento reflexivo-crítico, de aprendizaje colaborativo-cooperativo y de diseños de actividades auténticas ligadas a la experiencia y a los intereses de los alumnos. Dicho de otra manera, los ambientes y contextos deben convertirse en retos que representen el motor motivacional para tomar decisiones, proponerse metas, reflexionar sobre qué hacer, cómo hacerlo y qué y cómo evaluar el progreso (Torrano et al., 2017).

De este modo, la actividad docente, así como las estrategias metodológicas en el aula, deben orientarse a crear ambientes de aprendizaje interactivos, cooperativos, dinámicos y reflexivos, donde los alumnos sean protagonistas y agentes reflexivos en y desde su acción (Jonnaert et al., 2008; Kortagen et al., 2006).

\section{Principios clave para el diseño de acciones formativas para el fomento de la autorregulación}

En la línea de los estudios sobre el diseño de programas formativos para el desarrollo de competencias y aprendizajes más reflexivos, críticos, creativos y autorregulados (Correa, 2013; Jonnaert et al., 2008; Moya y Luengo, 2009; Tardif, 2008; Tejada y Ruiz, 2013), estos se centran en adoptar propuestas globalizadas e interdisciplinares a la hora de organizar los contenidos y un trabajo metodológico y evaluativo distinto. Lógicamente el papel y las funciones de los docentes y de los marcos organizativos también se ven afectados por este diseño de acciones formativas desde la lógica de las competencias y del desarrollo de aprendizajes reflexivo-críticos y autorregulados.

Coll (2014) señala la necesidad de trabajar en este enfoque de diseño formativo desde una perspectiva constructivista, sociocultural contextualizada que obliga a establecer un currículum que sepa diferenciar lo imprescindible de lo deseado. De este modo, el aprendizaje se debe focalizar en 
potenciar la reflexión crítica en y desde la acción, integrando contenidos, experiencias sociales y culturales que faciliten el empoderamiento y la transformación. Desde este planteamiento, se debe garantizar la implicación de situaciones de aprendizaje en las que el alumno se plantee retos, investigue, indague y resuelva circunstancias complejas conectadas con el mundo social actual, como resultado de la movilización del contraste de saberes y recursos a partir de la reflexión y la creación de conocimientos en grupos cooperativos. En el plano metodológico, estas ideas plantean nuevos retos a la hora de diseñar las estrategias de enseñanza y los sistemas de evaluación que permitan un seguimiento adecuado del proceso de aprendizaje.

En lo que respecta al papel y las funciones de los docentes, estos deben trabajar en equipo, necesariamente, para establecer propuestas interdisciplinares de organización de los contenidos, metodologías y estrategias que se concreten en prácticas cooperativas. Así, se espera una acción formativa con planteamiento de casos prácticos, simulados o reales, que hagan referencia a problemas actuales del contexto social y cultural. Además, con la utilización de estrategias que sitúen al alumno en una escena para autoevaluarse, reflexionar críticamente sobre su propio proceso de aprendizaje, pero sobre todo para crear y construir nuevos conocimientos a partir de la acción compartida y mediada entre docentes y compañeros.

Se deben asumir diseños formativos más flexibles, integradores de experiencias sociales y culturales, y de todo lo que supone la integración de la enseñanza formal, no formal e informal. Desde el punto de vista organizativo, se requiere de mayor flexibilidad en los agrupamientos y la configuración de los grupos de trabajo, para promover el desarrollo y la puesta en acción de estrategias cooperativas y personalizadas, de proyectos e itinerarios individualizados, de una exigencia en lo relativo a la apertura de los centros al entorno (familiar, social y cultural), de la construcción compartida del currículum, gracias a la cooperación e integración de escenarios (familia, escuela y comunidad).

\section{Objetivos}

Este artículo pretende discutir el modelo de patrones de aprendizaje de Vermunt $(1998,2005)$ y su influencia en el diseño y desarrollo de acciones formativas que garanticen experiencias en el contexto de educación primaria orientadas a potenciar un aprendizaje autorregulado. Para ello, se consideran las evidencias empíricas de los estudios recientes de Martínez-Fernández, Galera-Bassach y García-Orriols (2017) y Martínez-Fernández, 
García-Ravidá, García-Orriols y Martí-Garbayo (2018) en un centro educativo de Barcelona, España. En tal sentido, este artículo se plantea los siguientes objetivos:

» Describir los elementos clave del modelo de patrones de aprendizaje de Vermunt (1998, 2005).

» Definir bases y fundamentos de acciones formativas encaminadas al fomento de la autorregulación.

» Discutir el modelo de patrones de aprendizaje (Vermunt, 1998, 2005) para el diseño y desarrollo de acciones formativas orientadas a un aprendizaje autorregulado.

\section{Los patrones de aprendizaje y el diseño de acciones formativas}

Martínez-Fernández, Galera-Bassach y García-Orriols (2017) desarrollaron un estudio a partir del proyecto "Patrones de aprendizaje en los alumnos y profesorado de educación primaria y secundaria" que está llevando a cabo el Grupo de Investigación Pafiu, de la Universidad Autónoma de Barcelona, en la Escuela Mare de Déu de la Salut, Barcelona, España, en el cual se plantea un conjunto de acciones y resultados relevantes alrededor del modelo teórico de Vermunt (1998, 2005). En este, 172 alumnos desde cuarto a sexto grado de educación primaria de entre 9 y 12 años, durante los cursos 2014/15 (pretest) y 2015/16 (postest), respondieron la versión en línea adaptada para niños (Martínez-Fernández, García-Orriols y García-Ravidá, 2015) del Inventory of Learning Styles de Vermunt (1998).

La recogida de datos se caracterizó por una batería de instrumentos de carácter cuantitativo (Inventario de patrones de aprendizaje en educación primaria o ILP primaria, de Martínez-Fernández et al., 2015) y cualitativo (pautas de observación directa y entrevistas al profesorado), que contrastados, tienen la finalidad de identificar los patrones de aprendizaje en los estudiantes.

El primero, ILP primaria, está compuesto por 60 ítems que se agrupan en 16 subescalas, distribuidos en dos partes. Para responder este instrumento se utiliza una escala de Likert que va del 0 (Nunca/No) al 2 (Siempre/Sí). Estas 16 subescalas configuran los cuatro patrones de aprendizaje (MD, AD, RD y UD) propuestos en el modelo de Vermunt (1998, 2005). La primera parte del cuestionario pretende recoger datos en relación con las concepciones de aprendizaje y la orientación motivacional. La segunda, está dirigida a identificar las estrategias de procesamiento y la regulación. Los índices de confiabilidad de este inventario son $\operatorname{MD}(, 79), \operatorname{AD}(, 78)$, 
RD $(, 68)$ y UD $(, 62)$, y parecen ser consistentes tal como lo indica el estudio de Martínez-Fernández, García-Ravidá, García-Orriols y Martí-Garbayo (2018) en ciertos aspectos evolutivos en la etapa de transición de educación primaria a secundaria.

Por otro lado, se agregaron variables sociodemográficas como edad y género, además del autoconcepto académico como estudiante (Malo, Regular, Bueno y Excelente), el rendimiento escolar (nota media del curso) y la valoración del esfuerzo en una escala del 1 al 10. Para dar respuesta a este inventario, los estudiantes dispusieron de 60 minutos, aproximadamente, y estuvieron acompañados por un miembro del Grupo de Investigación Pafiu para resolver dudas que el conjunto de enunciados pudiera generar.

En lo referente a las pautas de observación directa, un grupo de expertos, con el soporte de una rúbrica de seguimiento que recogía enunciados sobre evidencias acerca de los componentes de aprendizaje (concepciones, motivaciones y estrategias de procesamiento y de regulación), realizó, por parejas, observaciones directas en las aulas. Así pues, cada pareja presenció cuatro sesiones de una hora con el tutor de cada grupo. Seguidamente, en grupos de discusión, se contrastaban los resultados para definir acuerdos interjueces, después se continuaba el mismo proceso de contrastación con los resultados obtenidos de la aplicación del inventario.

Por último, mediante reuniones de una hora con cada tutor de alumnos, un experto realizó una entrevista en cada grupo con el fin de recoger datos de cada uno de los estudiantes en relación con sus patrones de aprendizaje, para luego contrastar esta información con la obtenida en las observaciones y en el ILP, mediante grupos de discusión de expertos.

Una vez recogidos los datos y valoradas las apreciaciones y decisiones de los grupos focales, se realizó un conjunto de análisis estadísticos descriptivos y de fiabilidad mediante el programa sPSS.

Como resultado, en un primer momento (pretest), en el curso 2014/15 se identificaron, por un lado, sujetos orientados a la construcción de conocimiento, con interés personal y autorregulación (MD) pero, a su vez, con necesidad de estimulación y cooperación (UD), que fueron caracterizados como MD/UD. Otros, orientados a la reproducción de la información bajo una regulación externa o grupo RD. Otro grupo de alumnos concebía el aprendizaje como uso del conocimiento con interés vocacional y que activaban estrategias de procesamiento concreto o grupo AD; y finalmente, sujetos ambivalentes con características propias del patrón UD. En cambio, en un segundo momento (curso 2015/16) se identificaron los patrones de aprendizaje tal como los plantea el modelo de Vermunt (1998, 2005): MD, AD, RD y UD. Además, se encontró una fuerte relación entre el autoconcepto, 
el esfuerzo y el rendimiento académico y los patrones de aprendizaje; se evidenció un mejor rendimiento y mayor autoconcepto y esfuerzo con el patrón $\mathrm{MD}$, mientras que, para los alumnos caracterizados por un patrón UD, se halló todo lo contrario (Martínez-Fernández, Galera-Bassach y García-Orriols, 2017).

Considerando dicha categorización, se confeccionó una distinción del grupo de clase en tres partes: aquellos alumnos con mayor puntuación en patrones MD y AD; aquellos con mayor puntuación en RD y UD, y otros con puntuaciones medias en dichos factores. De este modo, se obtuvieron tres grupos diferenciados de alumnos. Estos grupos permitieron diseñar acciones formativas orientadas a la activación de procesos creativos, profundos y de carácter autorregulado mediante la cooperación entre individuos donde los niños del grupo RD/UD eran más protagonistas de su proceso de aprendizaje a partir de estas experiencias hacia entornos reflexivos y autorregulados; $y$ acompañados en auténticos grupos cooperativos por los niños más próximos a patrones MD/AD (Martínez-Fernández et al., 2017).

A raíz de ello, y teniendo en cuenta la relación significativa negativa entre el patrón UD y los factores personales de autoconcepto, esfuerzo y rendimiento académico, se llevó a cabo, en el mismo centro educativo, durante los cursos 2016/17 y 2017/18, el diseño y desarrollo de la asignatura optativa Desarrollo Personal y Aprendizaje, en la transición de la educación primaria a la secundaria (Martínez-Fernández, García-Ravidá, García-Orriols y Martí-Garbayo, 2018). Así pues, 27 alumnos de primero de Educación Secundaria Obligatoria, aquellos con mayores puntuaciones en patrón UD y con los niveles más bajos en los factores personales mencionados, formaron parte de esta iniciativa con el fin de desarrollar un proyecto personal relacionado con el mejoramiento de aspectos del desarrollo cognitivo, socioafectivo y psicomotor orientados a mejorar los procesos de aprendizaje y de autorregulación para aquellos alumnos con un mayor riesgo de exclusión tanto académica como social. Algunos de los datos obtenidos en el estudio de Martínez-Fernández et al. (2018) muestran que fomentar este tipo de actividades y experiencias de autorreflexión, autoevaluación y planificación activa procesos de aprendizaje autorregulado que garantizan cierta mejora tanto en el aprendizaje como en el rendimiento académico de estos alumnos. Cabe destacar que los autores hacen referencia a la necesidad de "mover" las concepciones y los motivos para aprender como componentes clave que se vinculan con el cambio en las acciones de aprendizaje (regulación y procesamiento), tal como defiende Vermunt (1998). 


\section{Discusión del modelo de patrones de aprendizaje de Vermunt y el diseño de acciones formativas}

A la luz de los datos de la investigación realizada en el contexto de la Escuela Mare de Déu de la Salut y la reflexión sobre los fundamentos clave de las acciones formativas, es conveniente señalar que estas, orientadas a desarrollar patrones de aprendizaje vinculados a la autorregulación, la autonomía y el pensamiento crítico y creativo, deberían caracterizarse por principios tales como:

Una enseñanza más inclusiva, que garantice una atención personalizada, de acuerdo con las capacidades, las estrategias, los ritmos, las actitudes, etc. de cada uno, pero también enseñar desde la diferencia y la equidad para lograr desplegar todo el potencial de los alumnos según sus características. Enseñar significa, desde esta lógica, brindar oportunidades de aprendizaje ricas, valiosas, auténticas, reflexivas, críticas y autorreguladas. Se partía de la premisa de que alumnos con patrones de aprendizaje RD y UD no podrán Ilegar a participar de forma óptima en actividades formativas reflexivo-críticas y autorreguladas, pero justamente hay que empezar a trabajar metodológicamente con ellos para otorgarles posibilidades de iniciar este camino. Para ello será imprescindible trabajar en grupos cooperativos heterogéneos, con una cuidada mediación docente y entre iguales.

En este sentido, estudios como los de Olave y Villarreal (2014) muestran cómo la participación en pequeños grupos heterogéneos mejora la motivación y el aprendizaje reflexivo-crítico. El hecho de cuestionarse, de dudar porque el otro me provoca, de hacerse preguntas, de responderlas, de reflexionar sobre las ideas, expresar razones y argumentos, ante y con los otros, activa procesos metacognitivos que favorecen un aprendizaje más reflexivo, crítico y profundo.

La utilización, por parte de los docentes, de actividades asentadas en el trabajo cooperativo en grupos heterogéneos contribuye también a mejorar los procesos de autorregulación, ya que los escenarios donde se interactúa entre iguales, se discute, discrepa, interpela, se diversifican las ideas, se toman decisiones compartidas, se generan ayudas, hay mediación entre compañeros, facilitan a los estudiantes involucrarse, de forma activa y personal, en su propio aprendizaje para llegar a ser más conscientes de este, base para generar aprendizaje crítico y creativo.

Hay que pensar, en la misma línea, que todo este proceso de construcción del aprendizaje, a partir de la interacción entre iguales, activa las relaciones socioafectivas que influyen de manera relevante en la significación 
y construcción del aprendizaje, pero también en los procesos motivacionales. Este trabajo es sumamente necesario cuando se trata con alumnos con patrones de aprendizaje RD y UD que requieren de la activación de una motivación intrínseca, basada en el interés personal, el afecto, el ánimo y el esfuerzo.

La interacción entre iguales, de alumnos RD/UD y MD/AD, en las cuales estos últimos actúan como mediadores de sus compañeros, y un trabajo docente en el que se propongan actividades en las que los alumnos deban llegar a acuerdos, a tomar decisiones compartidas y consensuadas, a distribuir tareas en función de las características personales y a analizar los aspectos positivos y mejorables de cada uno, produce un verdadero proceso de cooperación y aprendizaje compartido. En esta línea, las investigaciones de los procesos de regulación socialmente compartida (Arenas, 2017; Järvenoja, 2010; Panadero y Alonso-Tapia, 2014) aportan una nueva perspectiva en la regulación del aprendizaje. La interacción entre iguales, mediante una verdadera cooperación, de interacción recíproca, desde posicionamientos de igualdad, les permite compartir y construir el conocimiento gracias al establecimiento de objetivos comunes, de selección de estrategias negociadas, de distribución de roles, funciones y apoyos y de formas de regulación propias del grupo, sin necesidad de apoyos externos. Este tipo de regulación compartida y compleja (Järvenoja, 2010) conduce a un aprendizaje verdaderamente significativo, socialmente construido y profundo. Ahora bien, todos estos procesos requieren de un desarrollo de capacidades personales, de una madurez psicológica, de un control socioafectivo, de un nivel de conocimiento y aprendizaje y de un entorno social-familiar favorable que no siempre se da. Por lo tanto, habrá que analizar bien en qué momento y espacio se pueden desarrollar procesos formativos en los cuales el grupo autoorganice y autogestione su proceso de aprendizaje. Mientras tanto, habrá que ir estimulando los procesos de autorregulación y corregulación para llegar a una verdadera regulación socialmente compartida.

Una enseñanza diversificada y multivariada desde el punto de vista de las estrategias metodológicas. No es nada nuevo decir que cada persona tiene maneras distintas de aprender, de concebir el aprendizaje, de motivarse, de procesar la información y de relacionarse socialmente. El modelo de patrones de aprendizaje de Vermunt $(1998,2005)$ así lo constata. Por esta razón, desde el punto de vista de la enseñanza se deben diseñar metodologías diversas y multivariadas, que tengan en cuenta los tiempos (ajustar tiempos en función de los ritmos), los espacios, las tareas (comunes, personalizadas, autónomas, cooperativas) y el nivel cognitivo (complejas, sencillas, concretas, abstractas, deductivas, inductivas, etc.). 
De manera más concreta, las metodologías deberán respetar la adecuada relación entre experiencia-acción-contexto y, en este sentido, las modalidades centradas en la simulación, el análisis de casos reales, la resolución de problemas y el planteamiento de retos, entre otras, puede facilitar estas conexiones entre teoría, práctica y praxis. Asimismo es interesante plantear que no solo la ejecución de la tarea es importante, sino también la reflexión durante el proceso de ejecución de la tarea, más que su resultado (Ruiz et al., 2018). Otras iniciativas, como el pensamiento en voz alta a la hora de resolver un problema (Tesouro, 2005), el análisis e intercambio de materiales de elaboración personal y el estímulo por el cual los alumnos se hacen preguntas sobre los procesos y mecanismos que utilizan antes, durante y después de las tareas se convierten en estrategias facilitadoras para el desarrollo de pensamiento reflexivo-crítico. Torre (2008) plantea la idea de crear situaciones diversas para que los alumnos reflexionen críticamente y utilicen instrumentos para recoger dicho proceso de una manera sistematizada y consciente.

En la misma línea, siguiendo a Ruiz et al. (2018), la metodología interrogativa, como el arte de hacerse y responder preguntas, el diálogo reflexivo, el debate y la deliberación (Medina, Jarauta e Imbernón, 2010), representan estrategias que propician que los alumnos sean conscientes de su proceso de aprendizaje mediante el cuestionamiento de la teoría, de su conexión con las propias experiencias y de la reinterpretación y reconstrucción de los significados. En este sentido, la perspectiva narrativa y autobiográfica permite trabajar la reflexión en y sobre el proceso de construcción y desarrollo de competencias y profesional, fomentando que los alumnos generen reflexiones acerca de la conexión de sus experiencias personales y sus situaciones de aprendizaje (Alliaud y Suárez, 2011; Contreras, 2011; Suárez, 2011). En el campo educativo, la lectura reflexiva y crítica, los relatos, las autobiografías y las narrativas constituyen estrategias metodológicas significativas, ya que a través de ellas los alumnos no solamente describen los fenómenos, sino que también expresan las sensaciones de las personas que los componen (Suárez, 2013). Además, permiten que los alumnos descubran una nueva manera de explicar, integrando diversos espacios semánticos, géneros y formatos (Morales, 2010). La escritura y la narración ayudan a construir el pensamiento (Domingo y Gómez, 2015) relacionándolo con la acción, la experiencia y los aspectos afectivos y motivacionales (autoeficacia, planteamiento de metas propias, interés por las tareas) (Torrano et al., 2017). Por otra parte, las narrativas que incorporan la tecnología (Morales, 2010) —las cuales se componen de textos, imágenes, videos, sonidos, música y narración (McLellan, 2006) pueden representar otro estímulo para los alumnos, motivado por el hecho 
de romper con el esquema tradicional del texto escrito para pasar a un material audiovisual, lo que implica la reconstrucción del contenido en otro lenguaje y una apropiación de este (Badillo, 2012).

La responsabilidad y autonomía del alumno es indispensable. La clave está en crear espacios para trabajar esta autonomía y responsabilidad, propiciando y fomentando que los alumnos creen sus proyectos personales, tomen sus propias decisiones, reflexionen y argumenten de manera idiosincrática, con la adecuada mediación de los docentes y de los compañeros. En tal sentido, destacamos la línea de las recientes aportaciones de Martínez-Fernández et al. (2017) así como de Martínez-Fernández et al. (2018). Los mediadores (docentes), en este caso, deben facilitar claridad, concreción y realismo en las tareas y actividades planteadas. La falta de concreción y conexión de las actividades con los objetivos y resultados esperados puede representar un obstáculo para generar motivación, responsabilidad y compromiso (Ezzatti, 2016).

El trabajo cooperativo facilitará la interrelación entre los alumnos, y provocará conflictos sociocognitivos mediante el diálogo, el intercambio y la discusión de ideas y juicios. Es en este tipo de estrategias de trabajo cooperativo donde se puede fomentar la comunicación, el contraste de ideas, la argumentación compartida, la formulación de preguntas, las ayudas, la mediación a partir de la relación y tutoría entre iguales y, fundamentalmente, la reflexión compartida. Estos espacios cooperativos representan ambientes de mediación necesarios, imprescindibles para alumnos con patrones MD y UD, para la construcción significativa del conocimiento. Como plantean Zelaita y Camino (2018), "conviene propiciar un ambiente adecuado para la reflexión y exposición de argumentos, un clima que posibilite que los alumnos disfruten aprendiendo y desarrollen habilidades analíticas, prácticas, creativas y personales durante este proceso interactivo" (p. 201).

Las estrategias evaluativas, por la misma razón que las metodologías, deben ser diversas y variadas con una clara orientación hacia la evaluación formativa y formadora, combinando procesos de autoevaluación, heteroevaluación y coevaluación (Tejada y Ruiz, 2016) y desarrollando procesos efectivos de feedback y feed-forward como estrategia para la ampliación, reflexión, autoconciencia y mejora de los aprendizajes. En trabajos como los de Fabregat, Guàrdia y Forés (2016) se pone de manifiesto que dar feedback sobre cómo se desarrolla el proceso de construcción de los aprendizajes ayuda a los alumnos a mejorar los resultados. En otros estudios, como los de Ion, Silva y Cano (2013), los alumnos valoran positivamente el feedback formativo o feed-forward porque les permite ser más conscientes de las competencias desarrolladas. Estos procesos de 
feedback, realizados por uno mismo, mediante la autoevaluación o por otros (coevaluación), son fundamentales para analizar aciertos, errores y proponer mejoras (Fraile, Pardo y Panadero, 2018), para orientar la tarea, acompañar el proceso de desarrollo y construcción, supervisar el cumplimiento de las metas propuestas y proponer futuras acciones (Torrano et al., 2017). Concretamente, el uso de rúbricas para la autoevaluación y la coevaluación, las escalas de observación, los portafolios, blogs, entre otros, constituyen instrumentos facilitadores de estos procesos evaluativos.

\section{Conclusiones}

Después de discutir y hacer algunos aportes para la articulación de acciones formativas que contribuyan al desarrollo de patrones de aprendizaje orientados a la autorregulación, la autonomía y a un aprendizaje profundo, cabe decir, a modo de conclusión, que la enseñanza que promueve este tipo de aprendizaje debe caracterizarse por ser abierta al entorno, flexible y colaborativa entre distintos agentes educativos (profesores, alumnos, familia, equipos de asesoramiento, expertos educativos, entes de inspección, entidades locales, culturales y sociales). Allí, todos han de participar en la creación de proyectos educativos conjuntos, con una base colaborativa e innovadora.

Las comunidades de aprendizaje van a permitir trabajar de una manera interdisciplinaria para atender la diversidad de alumnos y ofrecer oportunidades a todos según sus patrones de aprendizaje, sus capacidades, intereses y contextos de procedencia. El reto actual está justamente en facilitar y mediar para que cada uno, desde la diferencia, pueda desarrollar todo su potencial a lo largo de la vida. En este sentido, si bien el objetivo es que los alumnos se autorregulen, generen pensamiento crítico para la construcción del conocimiento, no se puede asumir que todos ellos lo desarrollen en el mismo tiempo y espacio y con las mismas estrategias de aprendizaje. Por lo tanto, un alumno con un patrón RD/UD debe trabajar en espacios y tiempos de aprendizaje adecuados a sus características, empezando con una mayor presencia del docente y una mediación más clara sobre lo que ha de hacer y cómo lo ha de hacer (regulación externa) para, paulatinamente, ir abriendo espacio a un trabajo más personal, activo, reflexivo-crítico y responsable. En todo este proceso, el trabajo del docente y los equipos de asesoramiento son cruciales para mostrar una línea de trabajo consensuada, compartida y pertinente a las necesidades de cada uno.

Hay que seguir trabajando en la propuesta de diseños formativos desde una consideración socioconstructivista y situada (Coll, Mauri y Rochera, 2012; Panadero y Alonso-Tapia, 2014; Tejada y Ruiz, 2013; Torrano et al., 
2017), proponiendo actividades retadoras, con metas claras y precisas, con un nivel de logro accesible y adecuado a todos los alumnos. Es interesante que los objetivos sean propuestos por los estudiantes, que los discutan con todo el grupo y puedan decidirlos cooperativamente. En otros momentos, será pertinente que sean planteados por los docentes, pero, en este caso, habrá que trabajarlos y consensuarlos con los alumnos, ya que es clave que comprendan qué se espera de ellos y hasta dónde han de llegar. En este sentido, es importante programar actividades que generen un proceso de reflexión y toma de decisiones sobre qué espera cada estudiante de ellas, así como un objetivo personal de mejora.

Siguiendo con la argumentación, las actividades deben tener también un componente lúdico, que despierte motivación e interés, con propuestas de experimentación, de vivencias reales o simuladas, con la correspondiente reflexión y crítica, antes, durante y después de su realización. Hay que continuar creando espacios de valoración personal, en términos de autoevaluación, sobre las virtudes del propio trabajo y los aspectos débiles y por mejorar del proceso de aprendizaje. Pero también de la evaluación entre pares, que mediante procesos de coevaluación se ponen en situación de valorar al compañero de manera reflexiva y constructiva como mecanismo para seguir mejorando y creciendo. Las rúbricas de autoevaluación y de evaluación entre pares se convierten en instrumentos de valoración de este proceso, pero es preciso que estén construidas y consensuadas con los alumnos (Fraile et al., 2018).

Por otra parte, estas actividades retadoras deberán considerar una cuidadosa mediación de los factores emocionales y socioafectivos. Los docentes deben articular estrategias para realzar lo positivo de cada alumno, para animar y acompañar el proceso de aprendizaje, para crear expectativas positivas en cada uno, pero también para dinamizar el grupo de manera que se centre en cooperar y ayudar a los que más lo necesiten, para crear dinámicas en las que todos encuentren su lugar y su rol. Sería interesante que los estudiantes distribuyeran los roles y pudieran proponerlos con ayuda del docente.

Desde estas consideraciones, la enseñanza y el aprendizaje deben orientarse, sin lugar a dudas, hacia la personalización, la autonomía, la cooperación en grupos heterogéneos, la inclusión de todos, hacia un trabajo exhaustivo de mediación y orientación por parte de los profesionales, así como el apoyo y el trabajo colaborativo con todos los agentes educativos implicados.

Para finalizar, cabe anotar que los procesos de aprendizaje en alumnos de educación primaria y secundaria son transcursos lentos, donde intervienen otros factores, además de la escuela. Como consecuencia de 
ello, se considera importante realizar líneas de acción que traspasen las fronteras del aula o del propio centro educativo, abordando otros factores del entorno de estos alumnos, tales como sus familias, las cuales tienen un gran peso en su desarrollo integral.

\section{Referencias}

Alliaud, A. y Suárez, D. H. (coord.) (2011). El saber de la experiencia. Narrativa, investigación y formación docente. Buenos Aires: Clacso.

Arenas, K. A. (2017). Características de la regulación socialmente compartida de la escritura: aportes desde una experiencia investigativa. Lenguaje, 45(1), 35-60.

Badillo, M. E. (2012). Propuesta de comunicación y educación ambiental a través del Facebook y el uso de narrativas digitales. Entramado, 8(1), 128-139.

Berdonces Ballesteros, A. (2015). Relació entre els patrons d'aprenentatge dels mestres i la implicació familiar amb el patró d'aprenentatge dels nens (trabajo de grado). Universitat Autònoma de Barcelona, Barcelona, España. Biggs, J. (2010). Calidad del aprendizaje universitario. Madrid: Narcea.

Boekaerts, M. y Cascallar, E. (2006). How far have we moved towards the integration of theory and practice in self-regulation? Educational Psychology Review, 18(3), 199-210.

Boekaerts, M. y Corno, L. (2005). Self-regulation in the classroom: A perspective on assessment and intervention. Applied Psychology, 54(2), 199-231.

Bolívar, A. (2008). Didáctica y currículum: de la modernidad a la posmodernidad. Aljibe: Archidona.

Burbules, N. C. (2012). El aprendizaje ubicuo y el futuro de la enseñanza. Encounters, 13, 13-14.

Cladellas Pallarès, J. (2015). Patrons d'aprenentatge: La implicació de la família i el rol del mestres en l'aprenentatge d'alumnes d'onze i dotze anys a Catalunya (trabajo de grado). Universitat Autònoma de Barcelona, Barcelona, España.Coll, C. (2014). El sentido del aprendizaje hoy: un reto para la innovación educativa. Aula de Innovación Educativa, 232, 12-17.

Coll, C., Mauri, T. y Rochera, M. J. (2012). La práctica de evaluación como contexto para aprender a ser un aprendiz competente. Profesorado. Revista de Currículum y Formación del Profesorado, 16(1), 49-59.

Contreras, J. (2011). Experiencia, escritura y deliberación: explorando caminos de libertad en la formación didáctica del profesorado. InterAção, 38(1), 1-35. 
Correa, E. (2013). La profesionalización en contextos de formación inicial: ¿realidad o utopía? En Ruiz et al. (coords.), Formación para el trabajo en tiempos de crisis. Balance y prospectiva. Madrid: Tornapunta.

Cortés Giménez, E. (2015). Patrons d'aprenentatge segons I'edat: una aproximació amb alumnes d’Educació Primària a Catalunya (trabajo de grado). Universitat Autònoma de Barcelona, Barcelona, España.

De la Barrera, M. L., Donolo, D. y Rinaudo, M. C. (2010). Estilos de aprendizaje en alumnos universitarios: peculiaridades a la hora de aprender. Estilos de Aprendizaje, 6(6), 48-62.

Díaz Barriga, F. (2006). Enseñanza situada. Vínculo entre la escuela y la vida. México: McGraw-Hill.

Domingo, A. y Gómez, M. V. (2015). La práctica reflexiva. Bases. Modelos e instrumentos. Madrid: Narcea.

Efklides, A. (2009). The role of metacognitive experiences in the learning process. Psicothema, 21(1), 76-82.

Ezzatti, G. R. (2016). Aprendizaje autorregulado y mediación para la autonomía. Comunicaciones en Humanidades, 5, 70-75.

Fabregat, J., Guàrdia, L. y Forés, A. (2016). La perspectiva del profesorado respecto a la evaluación favorecedora del desarrollo de competencias. En E. Cano y M. Fernández (eds.), Evaluación por competencias: la perspectiva de las primeras promociones de graduados en el EEES. Barcelona: Octaedro-ICE UB.

Fraile, J., Pardo, R. y Panadero, E. (2018). Autoevaluación y autocalificación en el grado en ciencias de la actividad física y del deporte: estudio censal de las guías docentes. Profesorado: Revista de Currículum y Formación del Profesorado, 22(3), 163-182.

García Martín, P. (2014). Patrons d'aprenentatge i influència de la implicació familiar en nens catalans de nou anys (trabajo de grado). Universitat Autònoma de Barcelona, Barcelona, España.

Ion, G., Silva, P. y Cano, E. (2013). El feed-back y el feedforward en la evaluación de las competencias de alumnos universitarios. Profesorado. Revista de Currículum y Formación del Profesorado, 17(2), 283-301.

Järvenoja, H. (2010). Socially shared regulation of motivation and emotions in collaborative learning. Oulu: University Oulu.

Jonnaert, P. et al. (2008). La competencia como organizadora de los programas de formación: hacia un desempeño competente. Profesorado. Revista de Currículum y Formación del Profesorado, 12(3), $1-32$.

Korthagen, F. A., Loughranm, J. y Russell, T. (2006). Developing fundamental principles for teacher education programs and practices. Teaching and Teacher Education, 22(8), 1020-1041. 
Marambe, N. K., Vermunt, J. D. y Boshuizen, H. P. A. (2012). A cross-cultural comparison of student learning patterns in higher education. Higher Education, 64(3), 299-316.

Martínez-Fernández, J. R. (2002). Aprender: necesaria unión entre el querer, el saber y el poder. Revista de Pedagogía, 23(68), 477-494.

Martínez-Fernández, J. R., Galera-Bassachs y A., García-Orriols, J. (2017). Patrones de aprendizaje en educación primaria: identificación y acciones formativas inclusivas. Barcelona: Wolters Kluwer.

Martínez-Fernández, J. R., García-Orriols, J. y García-Ravidá, L. B. (2015). ILP primary school version. DOI: 10.13140/RG.2.2.27397.04329.

Martínez-Fernández, J. R. y García-Ravidá, L. B. (2012). Patrones de aprendizaje en estudiantes universitarios del Máster en Educación Secundaria: variables personales y contextuales relacionadas. Profesorado. Revista de Currículum y Formación del Profesorado, 16(1), 165-182.

Martínez-Fernández, J. R., García-Ravidá, L., García Orriols, J. y Martí Garbayo, L. (2018). Desarrollo personal y aprendizaje: desafíos a la escuela desde una mirada longitudinal a los patrones de aprendizaje. Contextos de Educación, 25, 54-66.

Martínez-Fernández, J. R. y Vermunt, J. D. (2015). A cross-cultural analysis of the patterns of learning and academic performance of Spanish and Latin-American undergraduates. Studies in Higher Education, 40(2), 278-295.

McLellan, H. (2006). Digital storytelling in higher education. Journal of Computing in Higher Education, 19(1), 65-79.

Medina, J. L., Jarauta, B. y Imbernón, F. (2010). La enseñanza reflexiva en la educación superior. Cuadernos de Pedagogía, 17, 4-42.

Morales, M. (2010). Hacia la democratización narrativa. Del hipertexto a la creación colectiva. Signo y Pensamiento, 29(57), 192-215.

Moya, J. y Luengo, F. (2009). Las competencias básicas en la práctica. Madrid: Proyecto Atlántida.

Olave, I. y Villarreal, A. C. (2014). El proceso de corregulación del aprendizaje y la interacción entre pares. Revista Mexicana de Investigación Educativa, 19(61), 377-399.

Ortega, F. (2017). Principios e implicaciones del nuevo modelo educativo. Revista Latinoamericana de Estudios Educativos, 47(1), 43-62.

Panadero, E. y Alonso-Tapia, J. (2014). Teorías de la autorregulación educativa: una comparación y reflexión crítica. Psicología Educativa, 20(1), 11-22.

Ruiz, C. et al. (2018). Creando contextos para el desarrollo del pensamiento reflexivo en la universidad. En Sabariego (coord.), El pensamiento reflexivo a través de metodologías narrativas: experiencias de 
innovación en educación superior. Cuadernos de Docencia Universitaria, 37. Barcelona: Octaedro-ICE.

Soler, E. (2006). Constructivismo, innovación y enseñanza efectiva. Caracas: Equinoccio.

Suárez, D. (2011). Narrativas, autobiografías y formación: una presentación y algunos comentarios. Educación y Pedagogía, 61(23), 11-24.

Suárez, D. (2013). Narrativas autobiográficas y formación en Argentina. La documentación narrativa de experiencias pedagógicas como estrategia de investigación-formación-acción entre docentes. Nuestra Idea. Revista de la Asociación del Magisterio de Santa Fe, 4, 50-65.

Tardif, J. (2008). Desarrollo de un programa por competencias. De la intención a su implementación. Profesorado. Revista de Currículum y Formación del Profesorado, 12(3), 1-16.

Tejada, J. y Ruiz, C. (2013). Significación del prácticum en la adquisición de competencias profesionales que permiten la transferencia de conocimiento a ámbitos propios de la acción docente. Profesorado. Revista de Currículum y Formación del Profesorado, 17(3), 91-110.

Tejada, J. y Ruiz, C. (2016). Evaluación de competencias profesionales en Educación superior: retos e implicaciones. Educación XXI, 19(1), 17-39.

Tesouro, (2005). La metacognición en la escuela: la importancia de enseñar a pensar. Educar, 35, 135-144.

Torrano, F., Fuentes, J. L. y Soria, M. (2017). Aprendizaje autorregulado: estado de la cuestión y retos psicopedagógicos. Perfiles Educativos, 39(156), 160-173.

Torre, J. C. (2007). Estrategias para potenciar la autoeficacia y la autorregulación académica en los estudiantes universitarios. En Prieto (coord.), La enseñanza centrada en el aprendizaje. Barcelona: Octaedro-ICE.

Vermunt, J. D. (1998). The regulation of constructive learning processes. British Journal of Educational Psychology, 68(2), 149-171.

Vermunt, J. D. (2005). Relations between student learning patterns, and personal and contextual factors and academic performance. Higher Education, 49(3), 205-234.

Vermunt, J. D., Bronkhorst, L. H. y Martínez-Fernández, J. R. (2014). The dimensionality of student learning patterns in different cultures. Learning patterns in higher education: Dimensions and research perspectives (pp. 33-55). Abingdon-Londres: Routledge.

Vermunt, J. D. y Endedijk, M. D. (2011). Patterns in teacher learning in different phases of the professional career. Learning and Individual Differences, 21, 294-302. 
Villar López, L. (2013). Perfiles de aprendizaje y rendimiento académico en niñas de Educación Primaria (tesis de maestría). Universidad Católica Andrés Bello, Caracas, Venezuela.

Zelaita, E. y Camino, I. (2018). El desarrollo del pensamiento crítico en la formación inicial del profesorado: análisis de una estrategia pedagógica desde la visión del alumnado. Profesorado. Revista de Currículum y Formación del Profesorado, 22(1), 197-214.

Zimmerman, B. J., Kitsantas, A. y Campillo, M. (2005). Evaluación de la autoeficacia regulatoria: una perspectiva social cognitiva. Evaluar, $5,1-21$. 